\title{
Hypertrophic pachymeningitis as an important neurological complication of granulomatosis with polyangiitis
}

\author{
Żaneta Smoleńska, Anna Masiak, Zbigniew Zdrojewski \\ Clinic of Internal Medicine, Connective Tissue Diseases, and Geriatrics, Medical University of Gdańsk, Poland
}

\begin{abstract}
Headache is a common symptom in patients with granulomatosis with polyangiitis (GPA) mainly due to chronic sinusitis or orbital disease. Meningeal involvement may thus remain unrecognized for a long time. This can lead to a significant delay in accurate diagnosis, serious local damage of the central nervous system and high relapse rates. New diagnostic techniques such as contrast MRI allow one to identify inflammation of the dura mater in the course of GPA more frequently. The objective of this article is to characterize hypertrophic pachymeningitis (HP) in patients with GPA and report diagnostic difficulties associated with this complication.
\end{abstract}

Key words: headache, hypertrophic pachymeningitis, granulomatosis with polyangiitis, diagnosis and therapy.

\section{Introduction}

Granulomatosis with polyangiitis (GPA), formerly known as Wegener's granulomatosis, belongs to the group of anti-neutrophil cytoplasm antibody (ANCA)-associated small vessel vasculitides (AAV). Granulomatosis with polyangiitis characteristically involves the upper and lower respiratory tract as well as the kidneys. However, this systemic inflammatory disease has a wider spectrum of clinical symptomatology, associated with heart, eyes, skin, joints and nervous system involvement. Depending on the disease extent, GPA is classified as either a generalized (systemic) phenotype characterized by the triad of vasculitis, pauci-immune focal necrotizing glomerulonephritis and necrotizing granulomatosis inflammation of the respiratory tract or a localized form with lesions limited to some organs: usually to the lung, middle ear, nasopharynx, throat and orbit. The latter form is more likely to develop in young, frequently female and ANCA negative patients and usually is not life-threatening, but often refractory to therapy [1].

Manifestations of nervous system involvement occur in both phenotypes of the disease, in $22-54 \%$ of patients with GPA [2-4]. Both the peripheral and the central nervous system (CNS) may be affected. Peripheral neurop- athies, mostly mononeuritis multiplex and rarely distal symmetric sensorimotor polyneuropathies, were reported as the dominant neurological presentations. Owing to the introduction of modern neuroimaging in diagnostic procedures, especially availability of MRI scans, the frequency of finding CNS involvement increased and nowadays it is observed in $4 \%$ to $13 \%$ of patients [5]. Central nervous system changes in GPA present various forms depending on the pathogenic background [6-8].

Three different histopathological patterns have been described: 1) vasculitis of the small vessels of the brain or spinal cord, 2) contiguous invasion of granuloma from nearby extracranial sites, and 3) isolated intracranial (cerebral or meningeal) granulomatous lesions [9]. In up to $30 \%$ of cases, neurological manifestations can be the result of a combination of the abovementioned lesions $[8,9]$. Patients with CNS involvement may present different neurological conditions including ischemic infarction $[10,11]$, intracerebral or subarachnoid hemorrhage [12], arterial and venous thrombosis, meningeal inflammations, cranial neuropathies due to nerve compression [13-15] and diabetes insipidus due to pituitary gland granulomatous changes [16].

\footnotetext{
Address for correspondence:

Żaneta Smoleńska, Clinic of Internal Medicine, Connective Tissue Diseases, and Geriatrics, Medical University of Gdańsk, 7 Dębinki St. 80-952 Gdańsk, Poland, e-mail: z.smolenska@wp.pl
}

Submitted: 4.09.2018; Accepted: 19.11.2018 
Meningitis is a rare neurologic complication of GPA, presenting mostly with a persistent headache. Because meningeal inflammation is usually detected in the dura mater and the leptomeninges (pia and arachnoid) are only sporadically affected, the exact nomenclature for this condition is hypertrophic pachymeningitis (HP), also termed as pachymeningitis (PM) or chronic hypertrophic pachymeningitis (CHP). Meninges of the brain and spinal cord can be affected. The presence of spinal cord pachymeningitis is less frequent but this form is significantly associated with relapsing/refractory diseases. Cerebral pachymeningitis tends to be associated with a better outcome than spine involvement and less often requires re-treatment [17]. Headache is a common symptom in patients with GPA mainly due to chronic sinusitis or orbital disease. Meningeal involvement may thus remain unrecognized for a long time [18]. This can lead to a significant delay in accurate diagnosis, serious local damage of the CNS and high relapse rates. The objective of this article is to characterize GPA associated with HP and report diagnostic difficulties associated with this complication.

\section{Epidemiology}

Hypertrophic pachymeningitis is an uncommon condition presenting as chronic fibrous inflammation of the dural meninx, which was first described by Charcot and Joffroy in 1869 as a cryptogenic thickening of the dura mater [19]. The exact mechanism underlying HP is still undefined. In half of cases HP is idiopathic, but in the other half it is immunologic. Infectious and histologic work-up could help to determine the etiology. HP may appear in GPA or other vasculitis, rheumatoid arthritis, Sjögren syndrome, lupus erythematodes, infections (syphilis, tuberculosis, fungi), sarcoidosis, IgG4-related disease and malignancy, mostly lymphoma [20, 21]. Therefore, accurate diagnosis and management of patients with GPA with suspected HP require exclusion of conditions other than ANCA-vasculitis leading to the dura mater thickening.

For a long time, HP has been considered as a very rare clinical complication of GPA. Nishino et al. [4] reported that only 2 of their 324 GPA patients showed dura mater thickening. Due to the introduction of new diagnostic tools such as contrast-enhanced magnetic resonance imaging of the brain (MRI), as well as greater knowledge and awareness, it is believed that GPA-associated HP is not as rare as previously thought. A study by Murphy et al. [22] reported that 11 of 19 GPA patients with neurologic symptoms showed meningeal thickening on brain MRI. Choi et al. [15] evaluated 65 patients with GPA; 25 of these had central nervous system involvement and HP was the second most common radiologic findings, following granulomatous disease before a stroke. Provenzale et al. [23] found meningeal thickening in the image of the brain in 3 of 15 GPA patients. A retrospective study of 35 patients with GPA and CNS involvement conducted by the French Vasculitis Study Group reported a 57\% incidence of HP. Cerebral pachymeningitis was noted in 16 cases and spinal cord pachymeningitis in 4 cases, while cerebral ischemic or hemorrhagic lesions were observed in 17 patients (49\%) [17].

\section{Pathogenesis}

It is thought that the pathogenic mechanism of pachymeningitis associated with GPA is an isolated granulomatous inflammation affecting only the meninges or, most frequently, the spreading of granulomatous tissue from the nasal, paranasal cavities or orbit and contiguously invasion to the adjacent structures including meninges [14]. Histopathological examination of biopsies showed dural thickening with fibrosis and some of them demonstrated granulomatous inflammation with geographic necrosis and multinucleated giant cells in the perivascular area. Small vessels in the meninges may be involved and the lumens of the veins are often occluded but morphological changes of the vessel wall are relatively slight [15]. In the above-mentioned early study done by de Luna pachymeningitis was classified as granulomatous phenotype G-CNS, mediated by the Th1 cytokine pathway.

\section{Clinical manifestations}

Hypertrophic pachymeningitis causes various neurologic symptoms associated with dural thickening, compression near the parenchyma, hindrance of sinus drainage or direct invasion of the cranial nerves. Involvement of the dura mater generates headache, which is the most common and almost always the first symptom (72.3\%) [15]. Headache is usually global, chronic, sometimes very severe, often refractory to analgesics or non-steroidal anti-inflammatory drugs and can be resolved with steroids. It should be also taken into consideration that some patients with GPA may have had non-localized or less prominent neurologic symptoms, such as unspecific mild headache, and that in this group it is important to investigate the clinical features or predicators of HP for early diagnosis and proper treatment. Hypertrophic pachymeningitis is usually an initial presenting manifestation of GPA rather than a later complication of disease, with up to $60 \%$ of HP presenting at disease onset [24].

Many observations conducted in several studies of patients with GPA indicate lower incidence of systemic manifestations such as pulmonary and renal involve- 
ment in patients with HP complications. In the Comite study [25] a strong association of HP with active localized disease was established $(70.3 \%$ of patients with HP had localized disease vs $10.8 \%$ who had early systemic disease and $18.9 \%$ who had generalized disease). Thus very careful examinations and modern neuroimaging should be considered for patients who present with unexplained headaches, especially in patients with paranasal involvement. Cranial neuropathies, seizures, cerebellar ataxia, encephalopathy and neuroophthalmologic complications are other symptoms which may develop in patients with HP [17]. Cranial nerve paralysis occurs in about $50 \%$ of these patients. Cranial nerves II, III, V, VI, VII, and $X$ are usually affected [22]. Some studies indicate that the optic nerve is the most frequently involved nerve (50\%) [15], while others suggest the fifth cranial nerve as the most commonly affected [7]. Ameliorating the function of optic and oculomotor nerves may lead to vision disturbances, diplopia and even blindness.

Some of the consequences of the inflammatory process in the dura mater are the hypercoagulability state and venous stasis caused by perifocal mechanical compression, which contribute to stenosis/occlusion and subsequent thrombosis of the venous sinus termed cerebral venous sinus occlusion and cerebral venous sinus thrombosis (CVSO/CVST). In the course of GPA and in idiopathic HP the superior sagittal sinus, bilateral transverse sinus, and sigmoid sinus appear to be the most frequently affected $[6,26]$.

There may be two explanations for this finding. First, these sinuses are next to the falx and tentorium, which are most frequently affected in HP patients. Second, as a systematic immunologic disease, the prethrombotic state of idiopathic hypertrophic cranial pachymeningitis (IHCP) tends to affect these sinuses. In patients with CVSO/CVST, the rate of occurrence of intracranial hypertension, severe headache, cerebral lesions, neurologic signs, seizures, behavioral symptoms, and cerebral herniation can be much higher compared with patients without CVSO/CVST [26]. It should also be noted that cerebral sinus thrombosis is one of the factors contributing to intracerebral hemorrhages but that these events occur extremely rarely in GPA patients with HP. Di Comite [25] suggested that active inflammation in the dura mater and vasculitis of small vessels in meninges may lead to spontaneous subdural hematomas (SDHs), as confirmed by Choi et al. [15] in their study on three patients.

\section{Diagnosis}

Early diagnosis and timely therapy may determine the course and prognosis, and most importantly, can prevent permanent neurological dysfunction.
Diagnosis of HP is based on characteristic neuroimaging findings in addition to pathology and the exclusion of secondary causes including infection, malignancy and other systematic autoimmune diseases. There are no specific abnormalities in laboratory tests regarding HP. In most cases inflammatory markers such as ESR and CRP are elevated. A positive serum ANCA, either cytoplasmic or perinuclear, is found in about twothirds of patients [18].

Hypertrophic pachymeningitis patients positive for anti-PR3 more often present systemic features of GPA including pulmonary and renal vasculitis. Anti-MPO positive patients are more likely to be older women with limited phenotype of GPA, and having less severe neurological involvement and lower levels of inflammatory markers such C-reactive protein [13]. Both CANCA and pANCA positive groups of patients have the same frequency of chronic sinusitis, otitis media, mastoiditis and retroorbital pseudotumor, and the same histopathological results. MRI scans in PR-3 positive patients are more likely to show parenchymal and leptomeningeal involvement. The measurement of PR-3 antibody level is not always useful in monitoring activity of HP in localized phenotype, because the PR-3 titer might not be elevated and relapses are localized only in the dura mater [25].

In most cases the examination of cerebrospinal fluid (CSF) shows nonspecific abnormalities such as mild pleocytosis, consisting mainly of lymphocytes and mild elevated protein concentration, but CSF analysis without abnormalities can also be seen [14]. Neuroimaging techniques such as computed tomography (CT) are characterized by relatively low sensitivity and insufficient specificity to allow detection of meningeal disease. The widespread application of MRI has greatly facilitated early recognition and follow-up of patients with HP. The definitions of HP require focal or diffuse thickening (> $2 \mathrm{~mm}$ ) and enhancement of dura mater on T1-weighted contrast-enhanced MRI [17].

The typical finding on MRI is seen in the dura mater, while leptomeninges involvement has been found less commonly. Two distinct MRI patterns have been described as follows: diffusely abnormal meninges unrelated to sinus or orbital disease and focal enhancing thickening adjacent to sinus or orbital disease [14]. Patterns of HP were described in terms of morphology (linear vs. nodular), symmetry, sites of involvement (falx, convexity, tentorium, and skull base), and concomitant invasion of pericranial structures $[17,26]$. Figure 1 shows extensive dural thickening at the left tentorium cerebelli on T1 coronal gadolinium-enhanced T1-weighted MRI in a patient with GPA.

Choi et al. [15] found in their retrospective analysis of 25 patients with GPA and CNS that among patients with GPA-related HP, the convexity $(n=8 ; 88.9 \%)$ was the 

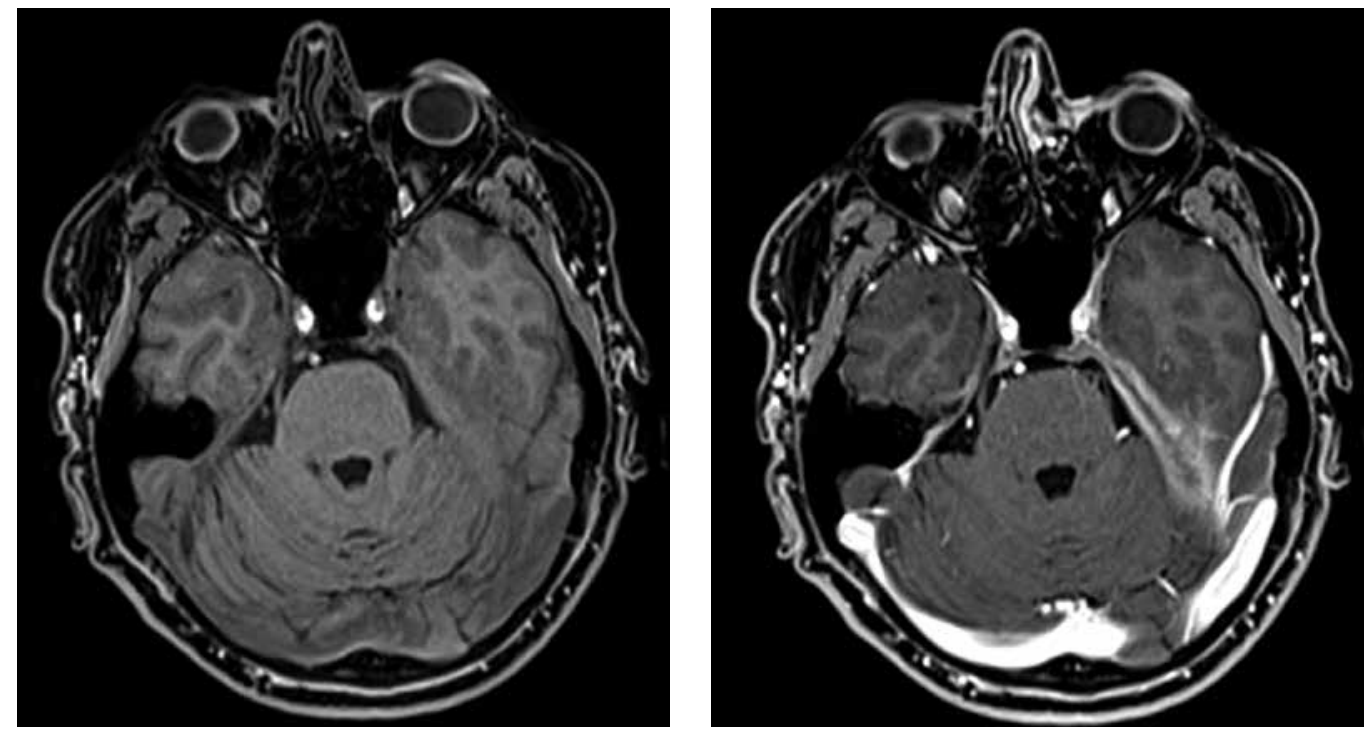

Fig. 1. Extensive dural thickening at the left tentorium cerebelli on T1 coronal gadolinium-enhanced T1-weighted MRI in patient with granulomatosis with polyangiitis from Department of Internal Medicine, Connective Tissue Diseases and Geriatrics, Medical University of Gdańsk, Poland.

most frequently involved site, and linear ( $n=8 ; 88.9 \%)$ and asymmetric $(n=6 ; 66.7 \%)$ brain MRIs were more common in patients with GPA-related HP. Reinhold-Keller et al. [6] confirmed that the falx cerebri and tentorium cerebelli are the most common sites of HP and the location around the cavernous sinus is a rare involvement. On axial post-contrast T1-SPGR images of the brain, thickening of dura mater in the falx cerebri and in the tentorium cerebelli has been described by radiologists as resembling "the Eiffel Tower at night" [19] (Fig. 2).
The problem with diagnosis of HP is due to nonspecific clinical manifestations including common headache and lack of specific laboratory tests. Radiological techniques show that dura mater thickening can be missed with the use of CT or because the sensitivity of normal contrast magnetic resonance imaging (MRI) is sometimes not sufficient, while venography for patients with $\mathrm{HP}$ is not routinely performed [22]. Because repeat brain MRI may show no or minimal radiological improvement despite clinical recovery as a result of residual postin-
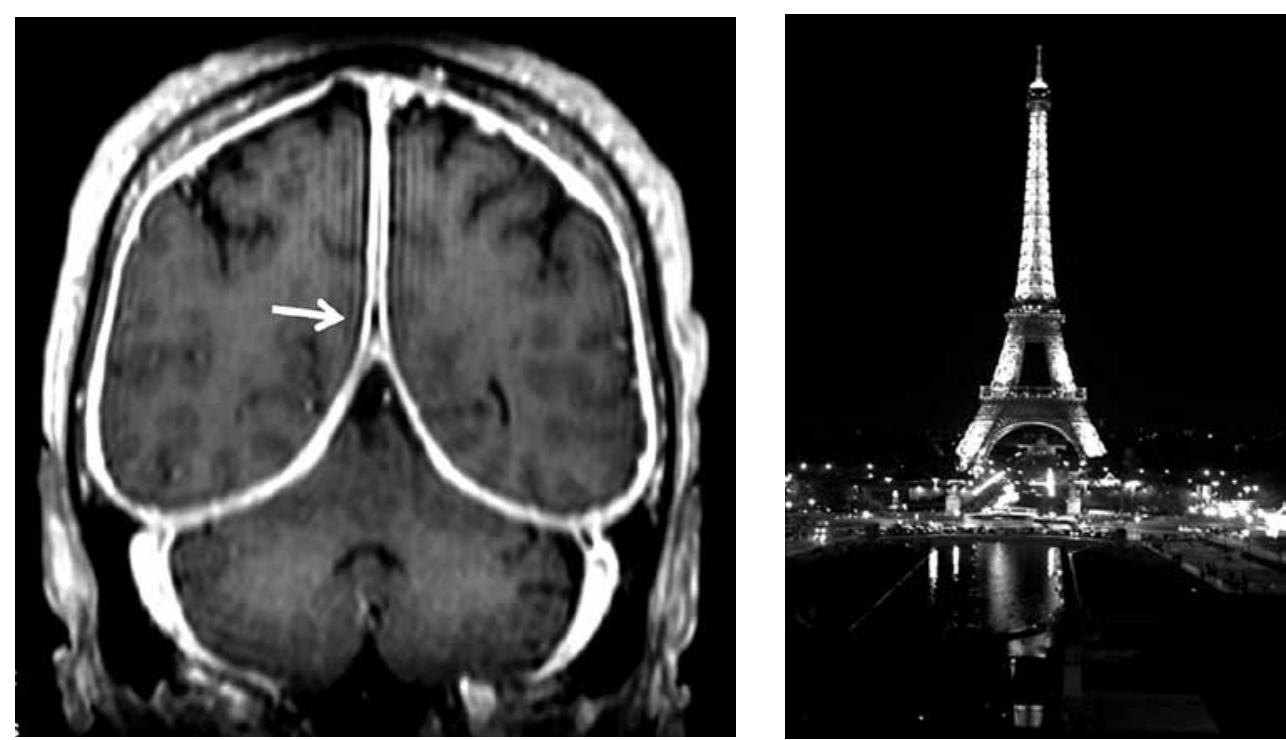

Fig. 2. Extensive dural thickening at the left tentorium cerebelli and falx cerebri on T1 axial gadoliniumenhanced T1-weighted MRI resembling Eiffel Tower at night [19]. 
flammatory fibrosis of the dura mater, regular MRI scans in monitoring HP are not proposed [18].

\section{Differential diagnosis}

Sarcoidosis is a granulomatous disorder which can involve virtually any organ, mostly the lungs, lymph nodes and skin. Nervous system involvement occurs in $5 \%$ to $15 \%$ of patients. The neurological manifestations include cranial neuropathy, with the facial and optic nerves being the most frequently affected, parenchyma lesions, hydrocephalus, aseptic meningitis, peripheral neuropathy, and myopathy. The majority of patients with neurosarcoidosis manifest chronic meningitis without a history of systemic sarcoidosis. The diagnosis can be difficult due to poor sensitivity of most diagnostic tests; however, in sarcoidosis with CNS involvement, the pia is changed more frequently than the dura and meningeal enhancement on MRI typically extends along the contour of the brain and within the brain sulci [27]. Gallium 67 scanning is a valuable imaging method for initial detection of systemic sarcoidosis, but it has limited utility because it is expensive and causes potential radiation exposure [28]. Definite diagnosis of neurosarcoidosis requires histopathological confirmation.

Primary dural tumors (e.g. meningioma or dural lymphoma) or secondary dural tumors (e.g. metastasis), should also be taken into consideration. The distinction between GPA and these conditions can be made based on associated clinical and radiological findings or by dural biopsy if the non-CNS manifestations of GPA are absent or subtle.

Erdheim-Chester disease is a rare disease, typically of middle age, characterized by abnormal multiplication of histiocytes. Infiltration of lipid-laden macrophages, multinucleated giant cells, lymphocytes and histiocytes occur in the bone marrow and cause generalized sclerosis of the long bones. Extraskeletal involvement occurs in about $50 \%$ of cases. Ataxia, exophthalmos, pituitary gland disease and HP are rare complications. Recently published reports from a multicenter French retrospective study of 60 patients with HP have not found any differences in demographics and neurological presentation among patients with idiopathic pachymeningitis, Erdheim-Chester disease and GPA [29].

Table I. Most common conditions leading to pachymeningitis - diagnostic differentiation

\begin{tabular}{|c|c|c|c|c|c|}
\hline & $\begin{array}{c}\text { Most } \\
\text { characteristic } \\
\text { organ } \\
\text { involvement }\end{array}$ & $\begin{array}{l}\text { CNS features apart } \\
\text { from HP }\end{array}$ & $\begin{array}{l}\text { Histopathological findings } \\
\text { in dura mater }\end{array}$ & $\begin{array}{l}\text { Laboratory } \\
\text { specific findings } \\
\text { or supportive } \\
\text { diagnostic tests }\end{array}$ & Therapy \\
\hline GPA & $\begin{array}{l}\text { ENT, lungs, } \\
\text { kidney }\end{array}$ & $\begin{array}{c}\text { Cranial neuropathy, } \\
\text { ophthalmoplegia, } \\
\text { cerebrovascular } \\
\text { events }\end{array}$ & $\begin{array}{l}\text { Necrotizing epithelioid } \\
\text { granuloma }\end{array}$ & $\operatorname{ANCA}(+)$ & $\begin{array}{l}\text { GKS, CYC, AZA, } \\
\text { MMF, MTX, RTX }\end{array}$ \\
\hline Sarcoidosis & $\begin{array}{l}\text { Lungs, hilar } \\
\text { adenopathy }\end{array}$ & $\begin{array}{c}\text { Facial nerve palsy, } \\
\text { leptomeningitis } \\
\text { mass lesions }\end{array}$ & $\begin{array}{c}\text { Noncaseating epithelioid } \\
\text { granuloma }\end{array}$ & $\begin{array}{c}\text { ACE } \\
1,25 \mathrm{OH} \mathrm{D3}\end{array}$ & $\begin{array}{l}\text { GKS, CYC, } \\
\text { TNFi, RTX }\end{array}$ \\
\hline $\begin{array}{l}\text { Infectious } \\
\text { meningitis }\end{array}$ & CNS & $\begin{array}{l}\text { Encephalopathy, } \\
\text { seizures }\end{array}$ & $\begin{array}{l}\text { Inflammatory cell infiltration } \\
\text { with neutrophils } \\
\text { predominance }\end{array}$ & $\begin{array}{l}\text { CSF analysis, urine } \\
\text { and blood culture }\end{array}$ & $\begin{array}{l}\text { Antibiotics, } \\
\text { tuberculostatic } \\
\text { and antifungal } \\
\text { agents }\end{array}$ \\
\hline $\begin{array}{l}\text { Erd-heim- } \\
\text { Chester } \\
\text { disease }\end{array}$ & $\begin{array}{c}\text { Skeletal, heart, } \\
\text { aorta }\end{array}$ & $\begin{array}{l}\text { Hypophysitis } \\
\text { cerebellar } \\
\text { dysfunction }\end{array}$ & $\begin{array}{c}\text { Xanthogranuloma with foamy } \\
\text { histiocytes }\end{array}$ & BRAF gene mutation & $\begin{array}{l}\text { Vemurafenib, } \\
\text { GKS }\end{array}$ \\
\hline $\begin{array}{l}\text { IgG4-related } \\
\text { disease }\end{array}$ & $\begin{array}{l}\text { Pancreas, sali- } \\
\text { vary gland }\end{array}$ & $\begin{array}{c}\text { Orbital } \\
\text { pseudotumor, } \\
\text { cranial neuropathies }\end{array}$ & $\begin{array}{l}\text { Lymphoplasmacytic infiltrates } \\
\text { with lgG4 cell predominance, } \\
\text { storiform fibrosis, and obliter- } \\
\text { ative phlebitis }\end{array}$ & Serum IgG4 & $\begin{array}{l}\text { GKS, AZA, } \\
\text { MMF, RTX }\end{array}$ \\
\hline $\begin{array}{l}\text { Idiopathic } \\
\text { pachymenin- } \\
\text { gitis }\end{array}$ & Absence & $\begin{array}{l}\text { Cranial neuropa- } \\
\text { thies, cerebellar } \\
\text { dysfunction }\end{array}$ & $\begin{array}{l}\text { Lymphoplasmacytic infiltrates } \\
\text { with fibrous proliferation }\end{array}$ & Absence & GKS, RTX \\
\hline
\end{tabular}

TNFi - TNF inhibitors; MTX - methotrexate; CYC - cyclophosphamide; GKS - glucocorticosteroids; AZA - azathioprine; ENT - ear-nose-throat; CNS - central nervous system, ANCA - antineutrophil cytoplasmic antibodies; RTX - rituximab; ACE - angiotensin converting enzyme; CSF-cerebrospinal fluid; MMF-mycophenolate mofetil 
Infectious meningitis (including tuberculosis) may cause thickening of the dura mater. Typically, in tuberculosis, the distribution of dural enhancement involves the basal cisterns, while in other infections clinical and biochemical examinations are helpful in the distinction between GPA and infectious meningitis. As with neurosyphilis, the distinction can often be reached by positive serologic VDRL or fluorescent treponemal antibody test results and by positive cerebrospinal fluid findings [30].

IgG4-related disease (IgG4-RD) represents a subset of cases previously diagnosed as idiopathic hypertrophic pachymeningitis. Inflammatory infiltrate, mainly consisting of $\mathrm{B}$ and $\mathrm{T}$ lymphocytes, activates fibroblasts and induces collagen deposition, leading to tissue hypertrophy and increased dural thickness. IgG-4RD in the brain parenchyma or spinal cord has never been reported and central nervous system involvement has been demonstrated in pituitary gland changes and as HP [31] IgG-4RD as a recently recognized disease is characterized by sclerosing lesions usually in the pancreas, salivary and lacrimal glands and is mostly associated with a raised serum IgG4 level and elevated IgG4 to IgG plasma cell ratio upon immunohistochemical staining [32]. MRI study and positron emission tomography with carbon 11-labeled methionine of the brain are preferred but biopsy with histopathological examination finally confirms the diagnosis. The most common conditions leading to pachymeningitis with diagnostic differentiation are shown in Table I.

\section{Treatment}

Treatment of ANCA-associated HP consists of administration of cyclophosphamide (CYC) or other immunosuppressives plus high-dose corticosteroid therapy (CS). Some studies show that combination therapy with cyclophosphamide demonstrates greater efficacy leading to fewer relapses than a corticoid-only regimen. Most patients initially receive standard therapy with CS and CYC either per os or intravenously on monthly coursers in doses similar to those used in generalized GPA, switching after the induction of remission to oral methotrexate (MTX), azathioprine (AZA), or mycophenolate mofetil (MMF) for remission maintenance [16, 20, $33,34]$. Evidence shows that in refractory GPA also with CNS involvement or in patients with severe manifestations of the disease such as pulmonary vasculitis or glomerulonephritis especially with relapsing courses, the CD20 selective inhibitor rituximab (RTX) is an effective treatment [20].

Some studies suggest, however, that granulomatous manifestations such as orbital granuloma and pachymeningitis were more refractory to rituximab than vascu- litis manifestations [35] and annual relapse rates for PR3 patients are higher than for MPO-positive disease [16].

\section{Conclusions}

Hypertrophic pachymeningitis is not such a rare complication of GPA as has been previously thought. New diagnostic techniques such as contrast MRI allow one to identify this condition more frequently. Hypertrophic pachymeningitis is associated mostly with localized phenotype of GPA, when both PR-3 and MPO antibodies are present. It is currently up for debate whether ANCA-associated HP is a single or heterogeneous group of diseases. HP with PR-3 antibodies appears to resemble systemic GPA with pachymeningeal involvement, whereas anti-MPO positive HP is more likely to be limited to the CNS, and relapses of the disease occur less frequently, in a manner analogous to patients with anti-MPO-positive renal-limited vasculitis. It is important to diagnose headache in patients with or suspected of GPA, especially where it is persistent and refractory, in order to diagnose HP and introduce accurate treatment to prevent progression of the disease.

The authors declare no conflict of interest.

\section{References}

1. Stone JH. Wegener`s Granulomatosis Etanercept Trial Research G. Limited versus severe Wegener's granulomatosis: baseline data on patients in the Wegener's granulomatosis etanercept trial. Arthritis Rheum 2003; 48: 2299-2309.

2. de Groot K, Schmidt DK, Arlt AC, et al. Standardized neurologic evaluations of 128 patients with Wegener granulomatosis. Arch Neurol 2001; 58: 1215-1221.

3. Anderson JM, Jamieson DG, Jefferson JM. Non-healing granuloma and the nervous system. Q J Med 1975; 44: 309-323.

4. Nishino H, Rubino FA, DeRemee RA, et al. Neurological involvement in Wegener's granulomatosis: an analysis of 324 consecutive patients at the Mayo Clinic. Ann Neurol 1993; 33: 4-9.

5. Fragoulis GE, Lionaki S, Venetsanopoulu A, et al. Central nervous system involvement in patients with granulomatosis with polyangiitis: a single-center retrospective study. Clin Rheumatol 2018; 37: 737-747.

6. Reinhold-Keller E, de Groot K, Holl-Ulrich K. Severe CNS manifestations as the clinical hallmark in generalized Wegener's granulomatosis consistently negative for anti-neutrophil cytoplasmic antibodies (ANCA). A report of 3 cases and a review of the literature. Clin Exp Rheum 2001; 9: 541-549.

7. Higuera-Ortiz V, Reynoso A, Ruiz N, et al. Pachymeningitis in granulomatosis with polyangiitis: case series with earlier onset in younger patients and literature review. Clin Rheumatol 2017; 36: 919-924. 
8. Seror R, Mahr A, Ramanoelina J, et al. Central nervous system involvement in Wegener granulomatosis. Medicine 2006; 85: 54-65.

9. David A, Drachman MD. Neurological complications of Wegener's granulomatosis. Arch Neurol 1963; 8: 145-155.

10. Sivakumar MR, Chandrakantan R. A rare case of stroke in Wegener's granulomatosis. Cerebrovasc Dis 2002; 13: 143-144.

11. Peng W, Wang X. Hypertrophic pachymeningitis and cerebral infarction resulting from ANCA-associated vasculitis. Neurol India 2012; 60: 424-426.

12. Takei H, Komaba Y, Kitamura H. Aneurysmal sub-arachnoid hemorrhage in a patient with Wegener's granulomatosis. J Clin Exp Nephrol 2004; 8: 274-278.

13. Yokoseki A, Saji E, Arakawa M, et al. Hypertrophic pachymen ingitis: significance of myeloperoxidase anti-neutrophil cytoplasmic antibody. Brain 2014; 137: 520-536.

14. Bruggemann N, Gottschalk S, Holl-Ulrich K, et al. Cranial pachymeningitis: a rare neurological syndrome with heterogeneous aetiology. J Neurol Neurosurg Psychiatry 2010; 81: 294-298.

15. Choi HA, Lee MJ, Chung CS. Characteristics of hypertrophic pachymeningitis in patients with granulomatosis with polyangiitis. J Neurol 2017; 264: 724-732.

16. Graf J. Central nervous system disease in Antineutrophil Cytoplasmic Antibodies-Associated Vasculitis. Rheum Dis Clin North Am 2017; 43: 573-578.

17. De Luna G, Terrier B, Kaminsky P, et al. Central nervous sys tem involvement of granulomatosis with polyangiitis: clinicalradiological presentation distinguishes different outcomes. Rheumatology (Oxford) 2015; 54: 424-432.

18. Sakellariou GT, Kefala N. Pachymeningitis_in granulomatosis with polyangiitis: a case report and a review of the literature. Case Rep Rheumatol 2013; 2013: 840984.

19. Waliszewska-Prosół M, Dziadkowiak E, Budrewicz S, et al. Idopatyczny przerost opony twardej - opis przypadku. Pol Przegl Neurol 2015; 11: 72-76.

20. Jang Y, Lee ST, Jung KH, et al. Rituximab Treatment for Idiopathic Hypertrophic Pachymeningitis. J Clin Neurol 2017; 13 155-161.

21. Kupersmith MJ, Martin V, Heller G, et al. Idiopathic hypertrophic pachymeningitis. Neurology 2004; 62: 686-694.

22. Murphy JM, Gomez-Anson B, Gillard JH, et al. Wegener gran ulomatosis: MR imaging findings in brain and meninges. Radiology 1999; 213: 794-799.
23. Provenzale JM, Allen NB. Wegener granulomatosis: CT and MR findings. AJNR Am J Neuroradiol 1996; 17: 785-792.

24. Holle JU, Gross WL. Neurological involvement in Wegener's granulomatosis. Curr Opin Rheumatol 2011; 23: 7-11.

25. Di Comite G, Bozzolo EP, Praderio L, et al. Meningeal involvement in Wegener's granulomatosis is associated with localized disease. Clin Exp Rheumatol 2006; 24 (Suppl 41): 60-64.

26. Huang $\mathrm{K}, \mathrm{Xu} \mathrm{Q}$, Zhan R, et al. Cerebral venous sinus thrombosis secondary to idiopathic hypertrophic cranial pachymeningitis: case report and review of literature. World Neurosurg 2017; 106: 1052.e13-1052.e21.

27. Hebel R, Dubaniewicz-Wybieralska M, Dubaniewicz A. Overview of neurosarcoidosis: recent advances. J Neurol 2015; 262: 258-267.

28. Hoyle JC, Jablonski C, Newton HB. Neurosarcoidosis: clinical review of a disorder with challenging inpatient presentations and diagnostic considerations. Neurohospitalist 2014; 4: 94101.

29. Mekinian A, Maisonobe L, Boukari L, et al. Characteristics, outcome and treatments with cranial pachymeningitis: A multicenter French retrospective study of 60 patients. Medicine (Baltimore) 2018; 97: e11413.

30. Yin LS, Kumar G, Vijayananthan A. Diffuse intracranial meningeal enhancement in Wegener's granulomatosis - A rare finding. Eur J Radiol Extra 2008; 67: 5-8.

31. Baptista B, Casian A, Gunawardena H, et al. Neurological Manifestations of IgG4-Related Disease. Curr Treat Options Neurol 2017; 19: 14.

32. Popkirov S, Kowalski T, Schlegel U, et al. Immunoglobulin-G4-related hypertrophic pachymeningitis with anti-neutrophil cytoplasmic antibodies effectively treated with rituximab. J Clin Neurosci 2015; 22: 1038-1040.

33. Arnaoutoglou MA, Xerras CG, Kalevrosoglou IK, et al. Headache Linked to Intracranial Hypertension and Hypertrophic Pachymeningitis as the Initial and Dominant Presentation of Granulomatosis With Polyangiitis. Case Report and Review of the Recent Literature. Headache 2018; 15: 1-7.

34. Dash GK, Thomas B, Nair M, Radhakrishnan A. Clinico-radiological spectrum and outcome in idiopathic hypertrophic pachymeningitis. J Neurol Sci 2015; 350: 51-60.

35. Costa C, Santiago T, Espirito-Santo J, et al. Pachymeningitis and cerebral granuloma in granulomatosis with polyangiitis: is rituximab a promising treatment option? Acta Reumatol Port 2017; 42: 82-87. 